\title{
Variación espacio-temporal de los gremios alimenticios de escarabajos fitófagos (Coleoptera: Scarabaeidae) en el Caribe colombiano
}

\author{
Spatio-temporal variation of food guilds of phytophagous beetles' (Coleoptera: \\ Scarabaeidae) in the Colombian Caribbean \\ (iD SANDY GARCÍA-ATENCIA ${ }^{1 *}$; iD GERMÁN AMAT-GARCÍA² $\dagger$
}

${ }^{1}$ Universidad del Atlántico, Puerto Colombia, Colombia.sandyga.01@gmail.com

${ }^{2}$ Universidad Nacional de Colombia, Bogotá, Colombia. gdamatg@unal.edu.co

\begin{abstract}
*Autor de correspondencia
Sandy García-Atencia, Universidad de Atlántico, Km 7 vía antigua a Puerto Colombia, Barranquilla, Colombia. sandyga.01@gmail.com

Citación sugerida

GARCÍA-ATENCIA，S. \& AMAT-GARCÍA, G. 2021 Variación espacio-temporal de los gremios alimenticios de escarabajos fitófagos (Coleoptera: Scarabaeidae) en el Caribe colombiano. Revista Colombiana de Entomología 47 (2): e10990. https://doi.org/10.25100/socolen. v47i2.10990
\end{abstract}

Recibido: 08-Feb-2021

Aceptado: 27-Oct-2021

Publicado: 17-Dic-2021

Revista Colombiana de Entomología ISSN (Print): 0120-0488

ISSN (On Line): 2665-4385

https://revistacolombianaentomologia.univalle.edu.co

Open access

(c) (i) (2) BY-NC-SA 4.0

Publishers: Sociedad Colombiana de Entomología SOCOLEN (Bogotá, D. C., Colombia) https://www.socolen.org.co

Universidad del Valle (Cali, Colombia)

https://www.univalle.edu.co

(C) 2021 Sociedad Colombiana de Entomología SOCOLEN y Universidad del Valle - Univalle
Resumen: En el Caribe colombiano además de las modificaciones de los paisajes por cambios de uso del suelo, los marcados periodos de sequías y lluvias inciden en la supervivencia de los organismos. Estos cambios modulan la oferta de recursos para escarabajos con amplios hábitos alimenticios. En este trabajo, se evaluó la variación espacio-temporal de gremios de escarabajos fitófagos en un fragmento de Bosque seco Tropical (Bs-T) y un cultivo aledaño en seis muestreos; en cada zona se colocaron cuatro trampas de luz entre las 18:00 y las 6:00 y se midieron las variables de temperatura, humedad y precipitación. En el bosque se recolectaron 5.184 individuos distribuidos en 19 especies, y en el cultivo 1.919 individuos pertenecientes a 16 especies de las subfamilias Dynastinae, Melolonthinae y Rutelinae. Se encontraron cuatro gremios: Rizo-Filófago (RF), Sapro-Caulófagos (SC), Sapro-Melífagos (SM) y Xilo-Filófagos (XF); el gremio predominante correspondió al RF con diez especies. El menor número de especies y gremios se capturaron en marzo, julio y agosto, mientras que mayo presentó la mayor diversidad de especies y número de gremios. Los mayores valores de la diversidad coinciden con el aumento de la precipitación en el área de estudio, lo que presentó correlación positiva con la riqueza y abundancia de especies en bosque ( $\mathrm{p}=0,77$ para los dos casos) y cultivo ( $p=0,84$ y $p=0,66$, respectivamente) durante mayo. Los gremios de escarabajos fitófagos fluctuaron en composición, riqueza y abundancia principalmente como respuesta a la estacionalidad climática, más que a las características de cada una de las áreas.

Palabras clave: Bosque Seco Tropical, departamento del Atlántico, diversidad, escarabajos pleurosticti, temporalidad.

Abstract: In the Colombian Caribbean, in addition to landscape modifications due to changes in land use, marked periods of drought and rainfall affect the survival of organisms. These changes modulate the supply of resources for scarabs with broad feeading habits. The spatio-temporal variation of scarab beetle guilds in a fragment of Tropical Dry Forest (Bs-T) and a surrounding crop was evaluated in six samplings; in each zone, four light traps were placed between 18:00 to 6:00 and temperature, humidity and precipitation variables were measured. In the forest 5,184 individuals distribuited in 19 species were collected, and in the crop 1,919 individuals belonging to 16 species of subfamilies Dynastinae, Melolonthinae and Rutelinae. The phytophagous scarab beetles from forest and crop were represented by four guilds: Rhizo-Phylophagous (RP), Sapro-Caulophagous (SC), Sapro-Meliphagous(SM) and Xilo-Philophagous (XP); the predominant guild corresponded to the RP with ten species. In forest and crop, the dominant guild was RF with ten species, and the least number of species and guilds were collected in March, July and August, while May presented the highest diversity of species and guilds. The above coincides with the increase of precipitation, that showed positive correlation with richness and abundance in forest $(p=0.77$, both cases) and crop $(p=0.84$ and $p=0.66$, respectively) during May. The guilds of phytophagous fluctuated in composition, richness, and abundance mainly in response to climatic seasonality, rather than the characteristics of each of the area.

Keywords: Tropical dry forest, department of Atlantico, diversity, pleurosticti beetles, seasonality. 


\section{Introducción}

La distribución espacial y temporal de los organismos se explica principalmente por los recursos disponibles para sus actividades vitales, por las interacciones que se dan entre ellos y por el régimen climático a través del tiempo (Zobel 1997); lo que define la manera como se estructuran las comunidades en los ecosistemas. Sin embargo, la transformación de los bosques es uno de los impactos ambientales que más consecuencias trae a la biodiversidad local, ya que genera cambios drásticos en los hábitats, alterando los atributos de los ensambles establecidos (Martino 2007).

En el departamento del Atlántico los bosques son sometidos a una gran presión antrópica por extracción de madera y uso agrícola (Martínez-Hernández et al. 2010). Debido a esto, las coberturas boscosas presentan cambios que propician la dominancia de pocas especies con mayores capacidades de soportar condiciones de perturbación y que desplazan a otros grupos de insectos que cumplen importantes roles ecológicos, tales como escarabajos fitófagos de la familia Scarabaeidae. La mayoría de sus integrantes, tienen hábitos fitófagos, y pocos son depredadores. Estos organismos se caracterizan por tener amplia variedad de funciones en los ecosistemas como degradadores de materia orgánica, consumidores primarios y secundarios, polinizadores, así como alimento de otros insectos y varios grupos de vertebrados (Morón 1994). Por lo tanto, en ambientes forestales tienen un impacto en el ecosistema tanto por su participación en los procesos de fragmentación y reciclaje de la madera y la hojarasca como por su posible papel como plagas (Morón 1985; Aragón et al. 2013).

En Colombia las investigaciones en este grupo se han incrementado los últimos años. Actualmente se cuenta con importante información sobre inventarios locales y nacionales (Restrepo-Giraldo et al. 2003; Suárez y Amat-García 2007; Gasca-Álvarez y Amat-García 2010; Sanabria-García et al. 2012; Vallejo y Wolff 2013; García-Atencia et al. 2015; García-Atencia y Martínez-Hernández 2015; López-García et al. 2015a, b) e identificación de especies y fenología de reconocidas plagas agrícolas (Pardo-Locarno et al. 2012; Vallejo y Wolff 2013).

Las investigaciones sobre diversidad de escarabajos fitófagos son escasas. Sin embargo, se resaltan los realizados por Alcázar-Ruíz et al. (2003), Reyes-Novelo y Morón (2005), García-de Jesús et al. (2016), Ibarra y Damborsky (2017) y Serrano y Barro (2017) para el neotrópico, y las investigaciones de García-Atencia et al. (2015) y Villalobos-Moreno et al. (2018) para Colombia. En los bosques secos del Caribe colombiano las contribuciones en este campo han obtenido importantes resultados para el entendimiento del papel de estas comunidades en los ecosistemas (Martínez-Hernández et al. 2010; Pardo-Locarno et al. 2012; García-Atencia et al. 2015; García-Atencia y Martínez-Hernández 2015). Si bien, García-Atencia et al. (2015) estudiaron previamente la variación de las comunidades de estos escarabajos en un fragmento de Bs-T, no tuvieron en cuenta la importancia de los policultivos como elementos que integran la matriz de estudio. Por lo tanto resulta necesaria la evaluación de la estructura de la comunidad en una matriz común en la región de estudio. Esto representaría un avance en el conocimiento ecológico de estas las comunidades y su comportamiento en un contexto de cambio por las condiciones naturales de ecosistema y antropogénico.
En el presente estudio se analizaron los cambios estacionales de los gremios de escarabajos fitófagos, en un bosque y un cultivo, y el cambio entre las comunidades de ambos tipos de vegetación, con el fin de aportar al conocimiento base para el uso y la conservación de los fragmentos de Bosque seco Tropical en el departamento del Atlántico.

Aunque los gremios de los Scarabaeidae fitófagos no están claramente descritos, la propuesta de Morón y Deloya (1991), modificada por Deloya et al. (2007), representa una contribución al entendimiento de cómo se diferencian las comunidades y cómo se asemejan en términos de fenotipo y de respuesta al ambiente (Colwell y Fuentes 1975). Es decir, debido a que las especies de una comunidad no están igualmente afectadas por los cambios en los ecosistemas, el análisis de gremios puede tener una mayor aproximación al entendimiento de respuesta de los organismos ante disturbios ambientales.

\section{Materiales y métodos}

Área de estudio. La Reserva Campesina de La Montaña -RCM- (o Tierra Arena), se encuentra ubicada en el municipio de Juan de Acosta, departamento del Atlántico, Colombia; localizada a $10^{\circ} 46^{\prime} 2,6^{\prime \prime} \mathrm{N}$ y $75^{\circ} 0,2^{\prime} 34^{\prime \prime} \mathrm{O}$, con altitudes entre 177 y 250 m (García-Atencia et al. 2015). La RCM forma parte del distrito Montes de María y Piojó, dentro de la región biogeográfica del Cinturón Árido Pericaribeño (Hernández-Camacho 1992). Los meses de mayor precipitación histórica corresponden a agosto y septiembre con promedios que van desde $100 \mathrm{~mm} / \mathrm{mes}$ hasta $580 \mathrm{~mm} / \mathrm{mes}$. Enero, febrero y marzo corresponden a los meses con menores precipitaciones, que van desde 0 hasta $30 \mathrm{~mm} / \mathrm{mes}$ y la temperatura oscila entre $\operatorname{los} 25^{\circ} \mathrm{C} \mathrm{y} 33^{\circ} \mathrm{C}$ (García-Atencia et al. 2015).

El presente estudio se realizó en un fragmento de Bs-T según lo descrito por Holdridge et al. (1971) y un policultivo adyacente separado a $1,5 \mathrm{~km}$ aproximadamente. El bosque, cuenta con una extensión de alrededor 47 ha y altitud máxima de $220 \mathrm{msnm}$. La vegetación predominante es la típica de BsT, descrita por Pizano y García (2014) en la que se presentan tres estratos vegetativos de dosel, arbustivo y sotobosque, y están presentes especies vegetales como Aphelandra pulcherrima (Jacq.) Kunth., Aspidosperma polyneron Müll. Arg., Astronium graveolens Jacq., Bursera simaruba (L.), Calycophyllum candidissimum (Vahl) DC., Capparis frondosa Jacq., Capparis odorattisima Jacq., Clavija sp., Hura crepitans L., Meliococcus oliviformis (Kunth), Ruelia macrophylla Vahl., Spondias mombin L., y Trichilia acuminata (Humb. \& Bonpl. Ex Roem. \& Schult.).

El policultivo, corresponde a un área de 30 ha con altura máxima de $241 \mathrm{msnm}$, dedicada a la siembra de Capsicum annuum L. (ají), Citrullus lanatus (Thunb.) Matsum. \& Nakai (sandía), Cucumis sativus L. (pepino), Cucurbita moschata Duchesne (ahuyama), Manihot esculenta Crantz (yuca), y Zea mays L. (maíz). También pueden encontrarse árboles de Crataeva tapia L. (naranjuelo), Bactris sp. (palmas de corozo), Maclura tinctoria (L.) (dinde), Platymiscium pinnatum (Jacq.) (trevol), y Sterculia apetala (Jacq.) H. Karst. (camajón), dispersos por el área a distancias que no sobrepasan los $30 \mathrm{~m}$ y cercas vivas de troncos de Gliricidia sepium (Jacq.) Kunth ex Walpers (matarratón), que rodean la zona.

Recolecta de individuos. Se realizaron seis muestreos entre 
marzo y agosto del 2013. Se colocaron ocho trampas de luz tipo embudo separadas aproximadamente $150 \mathrm{~m}$ entre sí, cuatro en bosque y cuatro en cultivo en forma de cuadrilátero para evitar el marcado efecto borde. Las trampas operaron una noche por mes desde las 18:00 hasta las 6:00, para un total de 72 horas de inversión y 48 muestras analizadas. Los días de recolecta se seleccionaron según el calendario lunar, lo que aseguro un muestreo nocturno de máxima oscuridad.

En cada área se tomaron las variables de temperatura y humedad ambiental con ayuda de Dataloggers marca GSI Super Quality. Para los valores de precipitación, se instaló un pluviómetro marca Hellman en el borde del fragmento, para obtener registros diarios de este parámetro en la RCM.

Fase de laboratorio. Los ejemplares recolectados se preservaron en etanol al $70 \%$ con su respectivo rótulo. Posteriormente, se determinaron con las claves propuestas por Endrödi (1985), Ramírez-Ponce y Morón (2009), Sanabria-García et al. (2012) y Vallejo y Wolff (2013). Adicionalmente, para cada especie se tomaron datos de longitud corporal para la estimación de la biomasa y se clasificaron según los gremios propuestos por Deloya et al. (2007). El material producto de este trabajo, está depositado en la Colección de Entomología del Instituto de Ciencias Naturales (ICN) en la ciudad de Bogotá.

Análisis de los datos. Se determinó la riqueza de especies y gremios capturados por muestreo y sitio; así como la abundancia (número de individuos) por especie. La biomasa se estimó como la cantidad de miligramos (mg) aportado por especies y gremios. Esta última fue calculada mediante la ecuación:

$$
\mathrm{B}_{\mathrm{e}}=N\left(0,305 * \mathrm{~L}_{*} 2,62\right)
$$

Donde, $B_{\mathrm{e}}$ es la biomasa en $\mathrm{mg}, N$ es la abundancia y $L$ es la media geométrica de la longitud corporal para las clases de tamaño relevantes. Se adoptaron las clases de tamaño citadas por Stork y Blackburn (1993) citadas en Sánchez y Amat (2005).

$$
\hat{\mathrm{C}} \mathrm{m}=\left(1-\frac{f_{1}}{\mathrm{n}}\left[\frac{(\mathrm{n}-1) f_{1}}{(\mathrm{n}-1) f_{2}+2 f_{2}}\right]\right) * 100
$$

Para evaluar la representatividad de los muestreos, se estimó la cobertura de la muestra $(\hat{\mathrm{C}} \mathrm{m})$ para cada sitio (Chao y Jost 2012) que es calculada por la fórmula:

Donde $\mathrm{n}$ es la abundancia de la muestra y $f_{1}$ y $f_{2}$ son los números de singletons (especies con un individuo en las muestras) y doubletons (especies con dos individuos en las muestras), respectivamente. Ésta, es una medida de completitud, dada la porción del total de individuos en una comunidad que pertenece a las especies representadas en la muestra (Chao y Jost 2012)

Se estimó la diversidad alfa, basada en la diversidad verdadera, donde: ${ }^{\circ} D$ es igual a la riqueza de especies, por lo que es insensible a las abundancias de las mismas; ${ }^{1} D$ (exponencial de Shannon), representa al número efectivo de especies de una comunidad teniendo en cuenta las abundancias de las especies comunes; y ${ }^{2} D$ (inverso de Simpson) tiene en cuenta las abundancias de las especies que dominan la comunidad (Jost 2006). Los valores se calcularon con el programa
iNEXT Online (Hsieh et al. 2020). Con estos se realizaron perfiles de diversidad por sitio y muestreos, acompañados de curvas de rango abundancia.

La diversidad beta fue estimada según lo propuesto por Carvalho et al. (2012), donde la disimilitud total $\left(\beta_{\mathrm{cc}}\right)$ incluye los componentes recambio de especies $\left(\beta_{-3}\right)$ y la disimilitud debida a las diferencias en la riqueza $\left(\beta_{\text {rich }}\right)$. Según este enfoque particionado, la máxima disimilitud se obtiene con un valor igual a 1 . Todo fue calculado con el paquete BAT de R desarrollado por Cardoso et al. (2015).

Se utilizó la prueba pareada no paramétrica de U Mann-Whitney para evidenciar si existían diferencias significativas entre abundancia de especies, biomasas de especies y abundancia y biomasa de gremios entre áreas. Así mismo una prueba de Kruskall-Wallis para saber si existían diferencias significativas entre la relación de la biomasa de especies y de gremios con el número de individuos en bosque y cultivo. Como complemento se realizaron correlaciones de Spearman entre las abundancias de gremios y las variables ambientales. Todo esto con ayuda del software R versión 1.25042 (Rstudio Team 2020).

\section{Resultados y discusión}

Se recolectaron 7.018 individuos agrupados en 23 especies pertenecientes a las subfamilias Dynastinae, Melolonthinae y Rutelinae y en cuatro gremios gremios: Rizo-Filófago (RF), Sapro-Caulófagos (SC), Sapro-Melífagos (SM) y Xilo-Filófagos (XF) (Tabla 1). La mayor riqueza la presentó la subfamilia Rutelinae con once especies, mientras que la mayor abundancia la presentó la subfamilia Melolonthinae con 5.180 especímenes. Las especies Phyllophaga pachypyga (Burmeister, 1855) y Astaena sp. aportaron un 29,2 \% y 29,6 $\%$ a la abundancia total, respectivamente. Para ambos usos del suelo estudiados, la cobertura del muestreo estuvo en el $99 \%$ de confiabilidad.

En el bosque se recolectaron 19 especies y 5.184 especímenes, y en el cultivo 16 especies y 1.919 individuos. La abundancia total de los fitófagos en las dos áreas no presentó diferencias significativas según la prueba pareada no paramétrica U Mann-Whitney $(\mathrm{U}=170 ; \mathrm{P}>0,05)$.

De acuerdo con los resultados obtenidos, el Bs-T estudiado alberga el 3,9 \% de los escarabajos fitófagos (Scarabaeidae) reportados para Colombia y el 41,7 \% de los descritos para el Caribe colombiano por Restrepo-Giraldo et al. (2003). Las altas abundancias se explican en gran medida por la presencia de Liogenys quadridens y P. pachypyga, de la subfamilia Melolonthinae y Paranomala inconstans de la subfamilia Rutelinae.

La mayor riqueza y abundancia la presentó el gremio RF con 16 especies y 6.897 individuos, respectivamente. Este gremio aportó el 97,5\% a la abundancia total de los gremios encontrados en la RCM. En bosque y en cultivo, el gremio predominante correspondió al RF con diez especies en cada sitio.

Los RF que, por su biología, pueden corresponder a potenciales plagas agrícolas, se caracterizan por tener estrategia de reproducción $r$ con gran tolerancia a las perturbaciones de los hábitats. Estas especies aprovechan los recursos que abundan en sistemas homogéneos, donde en los últimos años han predominado épocas prolongadas de sequía y perturbación antropogénica que induce a la homogeneidad en los recursos.

El aporte de D. dubius al gremio fue insignificante y sólo 
Tabla 1. Abundancia y biomasa (mg) de especies de los ensambles de escarabajos (Coleoptera: Scarabaeidae) fitófagos en bosque y cultivo de la RCM, departamento del Atlántico, Colombia. Abreviaturas: SM, Sapro-Melífagos; RF, Rizo-Filófagos; SC, Sapro-Caulófagos; XF, Xilo-Filófagos;

$\mathrm{S}$, riqueza; $\mathrm{N}$, abundancia.

\begin{tabular}{|c|c|c|c|c|c|}
\hline \multirow{2}{*}{ SUBFAMILIA-Tribu-Especies } & \multirow{2}{*}{$\begin{array}{l}\text { Bosque } \\
\text { Abundancia }\end{array}$} & \multicolumn{3}{|c|}{ Cultivo } & \multirow[t]{2}{*}{ Gremio } \\
\hline & & Biomasa & Abundancia & Biomasa & \\
\hline \multicolumn{6}{|l|}{ DYNASTINAE $N=104 ; S=6$} \\
\hline \multicolumn{6}{|l|}{ Cyclocephalini } \\
\hline Cyclocephala melanocephala (Fabricius, 1775) & 2 & 0,14 & 5 & 0,34 & SM \\
\hline Cyclocephala ovulum Bates, 1888 & 70 & 2,17 & 19 & 0,59 & SM \\
\hline Cyclocephala sp. 1 & 1 & 0,15 & 0 & 0,00 & SM \\
\hline Dyscinetus dubius (Olivier, 1789) & 0 & 0,00 & 2 & 0,06 & $\mathrm{RF}$ \\
\hline Euetheola humilis (Burmeister, 1847) & 1 & 0,15 & 0 & 0,00 & $\mathrm{SC}$ \\
\hline \multicolumn{6}{|l|}{ Pentodontini } \\
\hline Tomarus fossor (Latreille, 1833) & 2 & 0,31 & 2 & 0,31 & $\mathrm{SC}$ \\
\hline \multicolumn{6}{|l|}{ MELOLONTHINAE N=5.180; $S=6$} \\
\hline \multicolumn{6}{|l|}{ Macrodactylini } \\
\hline Barybas sp. & 12 & 0,17 & 0 & 0,00 & $\mathrm{RF}$ \\
\hline Liogenys quadridens (Fabricius, 1798) & 137 & 4,24 & 454 & 14,04 & RF \\
\hline \multicolumn{6}{|l|}{ Melolonthini } \\
\hline Phyllophaga menetriesi (Blanchard, 1851) & 108 & 35,75 & 0 & 0,00 & $\mathrm{RF}$ \\
\hline Phyllophaga impressipyga Frey, 1975 & 293 & 96,99 & 50 & 16,55 & RF \\
\hline Phyllophaga pachypyga (Burmeister, 1855) & 1036 & 158,13 & 1042 & 159,05 & $\mathrm{RF}$ \\
\hline \multicolumn{6}{|l|}{ Sericini } \\
\hline Astaena sp. & 2002 & 61,93 & 46 & 1,42 & RF \\
\hline \multicolumn{6}{|l|}{ RUTELINAE $N=1.734 ; S=11$} \\
\hline \multicolumn{6}{|l|}{ Anomalini } \\
\hline Paranomala inconstans (Burmeister, 1844) & 1279 & 195,22 & 137 & 20,91 & RF \\
\hline Paranomala sp. 1 & 96 & 14,65 & 27 & 4,12 & RF \\
\hline Paranomala sp. 2 & 3 & 0,09 & 1 & 0,03 & RF \\
\hline Paranomala sp. 3 & 0 & 0,00 & 38 & 2,58 & $\mathrm{RF}$ \\
\hline Paranomala sp. 4 & 7 & 0,22 & 79 & 2,44 & RF \\
\hline \multicolumn{6}{|l|}{ Geniatini } \\
\hline Leucothyreus sp. 1 & 3 & 0,20 & 0 & 0,00 & $\mathrm{XF}$ \\
\hline Leucothyreus sp. 2 & 41 & 2,79 & 0 & 0,00 & $\mathrm{XF}$ \\
\hline Leucothyreus sp. 3 & 0 & 0,00 & 3 & 0,09 & $\mathrm{XF}$ \\
\hline Leucothyreus sp. 4 & 0 & 0,00 & 1 & 0,03 & $\mathrm{XF}$ \\
\hline \multicolumn{6}{|l|}{ Rutelini } \\
\hline Pelidnota polita Latreille, 1811 & 2 & 0,66 & 0 & 0,00 & $\mathrm{XF}$ \\
\hline Xenopelidnota anomala (Burmeister, 1844) & 13 & 4,30 & 4 & 1,32 & $\mathrm{XF}$ \\
\hline Riqueza & 19 & - & 16 & - & - \\
\hline Total & 5108 & 578,26 & 1910 & 223,90 & - \\
\hline
\end{tabular}


estuvo presente en cultivo, hábitats donde se les ha recolectado como hospederos de plantas de maíz (Z. mays), arroz (Oryza sativa) y malanga (Xanthosoma sagittifolium) (Joly y Escalona 2010).

Variación espacial. Los mayores valores de la diversidad verdadera corresponden al bosque, mientras que el cultivo presentó un patrón similar con menor diversidad (Fig. 1). Los valores de las diversidades ${ }^{1} D$ y ${ }^{2} D$ reflejan la alta dominancia por pocas especies de la comunidad evidenciando que la estructura de los ensambles presentes en cada sitio se caracteriza por tener muchas especies con abundancias muy bajas y pocas especies dominantes. Por su parte $\beta_{\mathrm{cc}}$ arrojó una disimilitud de 0,58 , es decir que las especies no compartidas representan el $58 \%$ del número total de especies. De esto, el $45 \%$ se le atribuye al recambio en la riqueza de especies $\left(\beta_{-3}\right)$ que indica que ambos sitios presentan comunidades similares con pocas especies exclusivas; mientras el $13 \%$ a la diferencia en riqueza $\left(\beta_{\text {rich }}\right)$, que evidencia que hay pocas únicas de cada sitio.

La mayor abundancia y riqueza de escarabajos del Bosque, corresponde a la mayor heterogeneidad de recursos que permiten un mayor establecimiento y reproducción de las poblaciones. Por su parte, en el cultivo se presentan condiciones más homogéneas con permanencia muy limitada, pues los pobladores de la zona intercalan las áreas de cultivo cada año para evitar la pérdida en la calidad del suelo, lo que hace que sea aprovechado por aquellas especies capaces de explotar los recursos que allí se ofrecen garantizando su éxito reproductivo reflejado en sus altas abundancias. Sin embargo, aunque no existen diferencias significativas en la riqueza y la abundancia en los dos sitios, si se demuestra una diferencia en la composición de especies.
En el bosque Astaena sp., P. inconstans y P. pachypyga, del gremio RF, representan el $84 \%$ de la abundancia total mientras que E. humilis presentó el menor número de individuos. En el área del cultivo, las especies dominantes corresponden también al gremio RF y son L. quadridens, P. inconstans y $P$. pachypyga y (Fig. 2). Además, $P$. pachypyga comprende más del $50 \%$ del total con más de 1.000 individuos y sumado con L. quadridens y P. inconstans, representan el $85 \%$ de la abundancia total. Esta alta dominancia de especies con roles similares, pero con distintas abundancias en cada sitio, puede ser el resultado de hábitats degradados que, aunque albergan un número considerable de especies, las características intrínsecas de los sistemas y el estrés ambiental agravan las condiciones. Sin embargo, la estructura de la comunidad presenta diferencias entre hábitats.

El análisis no paramétrico, mostró que no hay diferencias significativas entre la biomasa de las especies en las áreas muestreadas $(\mathrm{W}=167 ; P>0,05)$. Aunque, el aporte de $P$. pachypyga a la biomasa en ambos sitios fue similar, siendo ligeramente mayor en el cultivo. Por su parte, Phyllophaga impressipyga $(\mathrm{U}=205,5 ; P<0,05)$, Astaena $\mathrm{sp}$. $(\mathrm{U}=140 ; P$ $<0,05)$, Paranomala sp. $3(\mathrm{U}=216 ; P<0,05)$ y $P$. menetriesi $(\mathrm{U}=204 ; P<0,05)$ presentaron diferencias significativas en cuanto a la biomasa aportada en cada sitio. En cuanto a las especies que dominaron por biomasa, se encontraron para el bosque Phyllophaga sp. 1 y P. inconstans, y para el cultivo, Phyllophaga sp. 1, siendo estas especies las que aportaron más de $150 \mathrm{mg}$ en cada hábitat. Con estos datos, se observó que algunas de las especies abundantes, no dominaron en términos de biomasa.

Con respecto a la biomasa de los gremios de Scarabaeidae fitófagos, los valores no presentaron diferencias significativas entre bosque y cultivo ( $\mathrm{W}=1,5 ; P>0,05)$ (Fig. 3$)$. Todos los

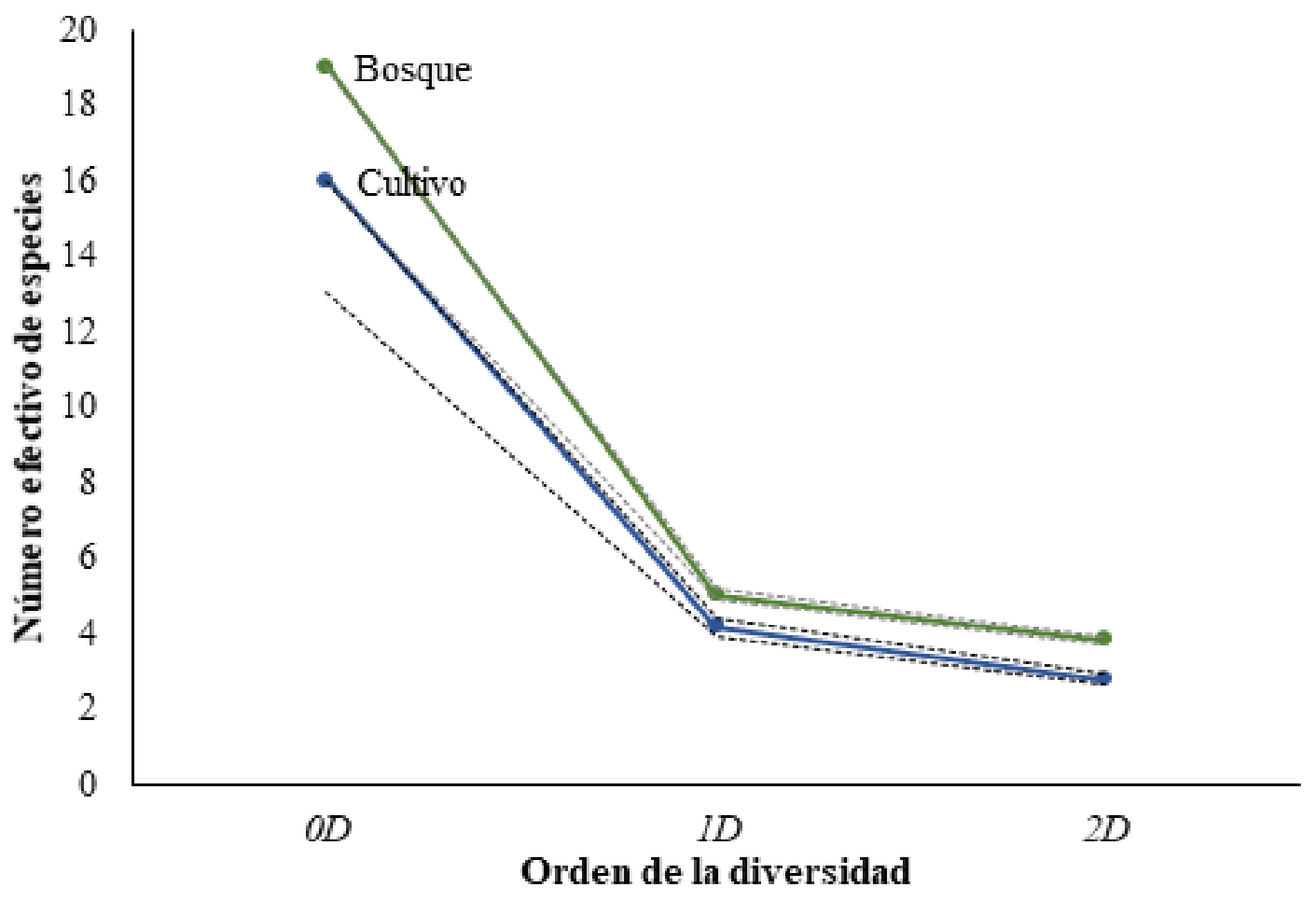

Figura 1. Perfil de diversidad de escarabajos fitófagos (Coleoptera: Scarabaeidae) para bosque y cultivo de la RCM. Líneas punteadas indican intervalos de confianza. 
gremios recolectados en el presente estudio se registraron en las dos áreas muestreadas, con mayor biomasa en el Bosque. Los RF en ambas áreas se caracterizaron por ser los que más aportaron a la biomasa, seguido por los XF. El aporte de los $\mathrm{SM}$ y SC fue menor de $1 \mathrm{mg}$. Este último presentó un aporte insignificante con respecto a los demás.

Para el bosque, la biomasa está dominada por P. inconstans que además de ser abundante presentó tallas mayores a 15 $\mathrm{mm}$. Esta especie ha sido considerada invasora que frecuenta pastizales inducidos y cultivos de gramíneas (Ramírez-Salinas et al. 2004). Por su parte en el cultivo, L. quadridens, presentó la mayor biomasa, por lo que la ausencia y/o poca abundancia de especies de tallas más grandes representaron este hábitat donde la vegetación es homogénea, el estrato arbóreo está ausente y los arbustos muy separados entre sí. Adicionalmente, las especies de talla pequeña tienen un metabolismo más rápido, lo que las obliga a estar más concentradas en los sitios que brindan recursos energéticos de manera permanente. Lo anterior refleja que aunque ambos sitios presenten condiciones de perturbación, en la comunidad de insectos fitófagos del cultivo abundan las formas pequeñas, o bien "oportunistas" (Clarke y Warwick 2001).

En cuanto a la relación entre la biomasa de gremios y el número de individuos, no se presentaron diferencias significativas en el bosque $(\mathrm{H}=1,33 ; P>0,05)$ ni en el cultivo $(\mathrm{H}$ $=2,08 ; P>0,05)$. Sin embargo, existen diferencias significativas entre la biomasa de especies y la abundancia de las mis- mas, tanto en bosque $(\mathrm{H}=5,08 ; P<0,05)$ como en el cultivo $(\mathrm{H}=7,78 ; P<0,05)$.

En el hábitat de Bs-T, Cyclocephala spp., T. fossor y $E$. humilis se recolectaron en mayo y junio, pero la baja garantía de supervivencia dada por las condiciones ambientales no permitió el establecimiento de los gremios SM y SC. En el caso de E. humilis, Bernardi et al. (2008) lo reportaron para Brasil como plaga de cultivos de arroz, caña de azúcar, millo y eucalipto, al igual que $T$. fossor que ha sido encontrado en capítulos y raíces de arroz, caña de azúcar, girasol, y maíz (Escalona y Joly 2006). Algunas especies de Cyclocephala son frecuentes en inflorescencias de las Araceae que se caracterizan por presentar espata y espádice, lo que las hace atractivas y ventajosas para el resguardo de algunas especies; por otro lado, les brindan temperatura estable, alimento, refugio, posibilidad de hallazgo de pareja, retribuyéndoles con su condición de polinizadores (Morón et al. 1997; Gasca-Álvarez, 2013). Otras especies del género pueden estar relacionadas con flores actinomorfas y frutos de una gran variedad de plantas de la familia Araceae que pueden estar presentes en variados tipos de ecosistemas (Milet-Pinheiro et al. 2017). Sin embargo, las especies de estos tres géneros de escarabajos tienen en común que, aunque sus hábitos son poco conocidos, sus larvas podrían ayudar a la mineralización y algunos adultos a la polinización (Morón et al. 1997).

Según Morón (2004), la mayor parte de las especies de escarabajos edafícolas deben estar adaptándose a las con-



Figura 2. Curvas rango-abundancia en Bosque y cultivo de la RCM. Abreviaturas: Asta, Astaena; Pinc, Paranomala inconstans; Phpac, Phyllophaga pachypyga; Phimp, Phyllophaga impressipyga; Lqua, Liogenys quadridens; Xanom, Xenopelidnota anomala; Ehum, Euetheola humillis; Pasp4, Paranomala sp. 4; Ddub, Dyscinetus dubius; Pasp2, Paranomala sp. 2. 
diciones de ecotono y de agro-ecosistemas, siendo también probable el aumento de especies originalmente asociadas a ambientes silvestres que puedan adaptarse a condiciones de monocultivo y que, por determinadas circunstancias ecológicas, puedan causar daños a las plantas de importancia económica. Por otro lado, la composición de la entomofauna usualmente se ve influenciada por las modificaciones en los hábitats naturales resultado de las actividades humanas, que propician la disminución de algunas especies y el éxito de otras a nivel local y regional.

Variación temporal. Los escarabajos (Coleoptera: Scarabaeidae) fitófagos en los hábitats estudiados presentaron una marcada estacionalidad distribuida con baja densidad y corroborada por la correlación positiva de la riqueza y abundancia de especies y gremios con la precipitación; esto se refleja en los valores más altos de abundancia concentrados en el muestreo de mayo, en el que la intensidad de las lluvias se incrementó (Tabla 2). En general, la prueba de correlación de Spearman mostró una correlación positiva entre la precipitación con la riqueza y abundancia en bosque ( $p=0,77$ para los dos casos) y cultivo ( $p=0,84$ y $p=0,66$, respectivamente). El comportamiento de la temperatura y la humedad fue similar para los dos sitios durante el periodo muestreado.

Este patrón es similar a lo encontrado por García-Atencia et al. (2015) en el mismo lugar, en diferentes localidades de Colombia (Martínez-Hernández et al. 2010; Útima y Vallejo 2010) y en países como México (Alcázar-Ruiz et al. 2003; Reyes-Novelo y Morón 2005; Ramírez-Ponce y Morón 2009; Lugo-García et al. 2011), Puerto Rico (Martínez et al. 2009) y en la Florida, EE.UU. (Buss 2006). En estos estudios, se ha encontrado una estrecha relación entre la riqueza y abundancia con el inicio del período de lluvias, lo que sustenta la diversidad dada por los índices usados en este trabajo durante mayo y junio. Es en estos meses algunas especies salen de sus cámaras pupales, como el caso de los adultos de Phyllophaga spp. que se reproducen y aumentan sus actividades, por lo cual son comúnmente conocidos como los "cocuyos de mayo" (Morón 1994).

Para el bosque, los mayores valores de diversidad ${ }^{0} D,{ }^{1} D$ $\mathrm{y}^{2} D$ se presentaron durante mayo que coincide con un ligero aumento de la precipitación, momento en el cual probablemente se incrementaron los recursos a explotar. Aunque los dos hábitats no presentaron diferencias en cuanto a ${ }^{0} D$, en el cultivo, los mayores valores de ${ }^{1} D$ y ${ }^{2} D$ se registraron para M4 (Fig. 4). Los menores valores de diversidad se presentaron en el M5 y M6 en los que se presentaron valores bajos de precipitación. Esto refleja las pocas abundancias recolectadas en los hábitats, y en el caso particular del bosque, la no obtención de individuos. Aunque ambos sitios presentan patrones similares, la composición y abundancia de las especies es diferente, lo que quiere decir que hay una variación bosque-cultivo, que corresponde a un posible reemplazo de los taxones que cumplen funciones ecológicas equivalentes. Estos cambios se presentan cuando existen perturbaciones intensas o cambios radicales en el uso del suelo, dando lugar a la introducción de nuevos integrantes (tal vez generalistas o con amplio espectro ecológico), al cambio en el dominio de especies presentes e inclusive a la desaparición de algún taxón (Morón 2001).

Según beta temporal total $\left(\beta_{c c}\right)$, en el bosque los muestreos fueron altamente disimiles entre ellos, teniendo valores por encima del $85 \%$ de disimilitud. Al aparecer las lluvias en mayo, en los BsT producen la emergencia de plántulas y demás recursos verdes que constituyen la principal fuente de alimentos de los escarabajos fitófagos (Scarabaeidae). Sin embargo, la disminución abrupta y atípica de la precipitación impide el normal paso a una nueva temporada lluviosa en estos ecosistemas, lo que disminuye la calidad y cantidad de los



Figura 3. Aporte de la biomasa en miligramos de los gremios de escarabajos fitófagos (Coleoptera: Scarabaeidae) en bosque y cultivo de la RCM, departamento del Atlántico, Colombia. Abreviaturas: RF, Rizo-Filófagos; XF, Xilo-Filófagos; SM, Sapro-Melífagos; SC, Sapro-Caulófagos. 
Tabla 2. Variación temporal de la abundancia de gremios de escarabajos fitófagos y variables ambientales en bosque y cultivo de la RCM. RF, Rizo-Filófagos; SC, Sapro-Caulófagos; SM, Sapro-Melífagos; XF, Xilo-Filófagos; Temp., Temperatura; HR, Humedad Relativa; Precip., Precipitación.

\begin{tabular}{ccccccccc}
\hline Sitio & Muestreo & RF & SC & SM & XF & Temp $\left({ }^{\circ} \mathbf{C}\right)$ & HR (\%) & Precip. (mm) \\
\hline & M1 & 67 & 1 & 0 & 0 & $25,15 \pm 2,1$ & $75,6 \pm 0,5$ & 8 \\
\cline { 2 - 9 } Bosque & M2 & 869 & 2 & 0 & 25 & $27,3 \pm 2,1$ & $75,6 \pm 7,3$ & 63 \\
\cline { 2 - 9 } & M3 & 3644 & 0 & 72 & 34 & $26,8 \pm 0,3$ & $83,8 \pm 1,6$ & 78 \\
\cline { 2 - 9 } & M4 & 381 & 0 & 1 & 0 & $26,4 \pm 0,7$ & $92,3 \pm 2,9$ & 99 \\
\hline & M5 & 12 & 0 & 0 & 0 & $26,5 \pm 0,1$ & $89,8 \pm 3,9$ & 21 \\
\hline \multirow{5}{*}{ Cultivo } & Motal & 4973 & 3 & 73 & 59 & - & - & - \\
& M1 & 16 & 0 & 0 & 0 & $25,15 \pm 0,3$ & $76 \pm 0,5$ & 8 \\
\cline { 2 - 9 } & M2 & 885 & 1 & 0 & 1 & $29,6 \pm 0,6$ & $69,2 \pm 1,8$ & 63 \\
\hline & M3 & 770 & 1 & 21 & 3 & $26,3 \pm 0,3$ & $87,0 \pm 1,9$ & 78 \\
\hline & M4 & 203 & 0 & 1 & 4 & $25,9 \pm 0,8$ & $92,2 \pm 4,0$ & 99 \\
\hline & M5 & 1 & 0 & 1 & 0 & $25,8 \pm 0,7$ & $91,3 \pm 4,4$ & 21 \\
\hline & M6 & 1 & 0 & 1 & 0 & $25,0 \pm 0,5$ & $92,7 \pm 2,4$ & 6 \\
\hline
\end{tabular}

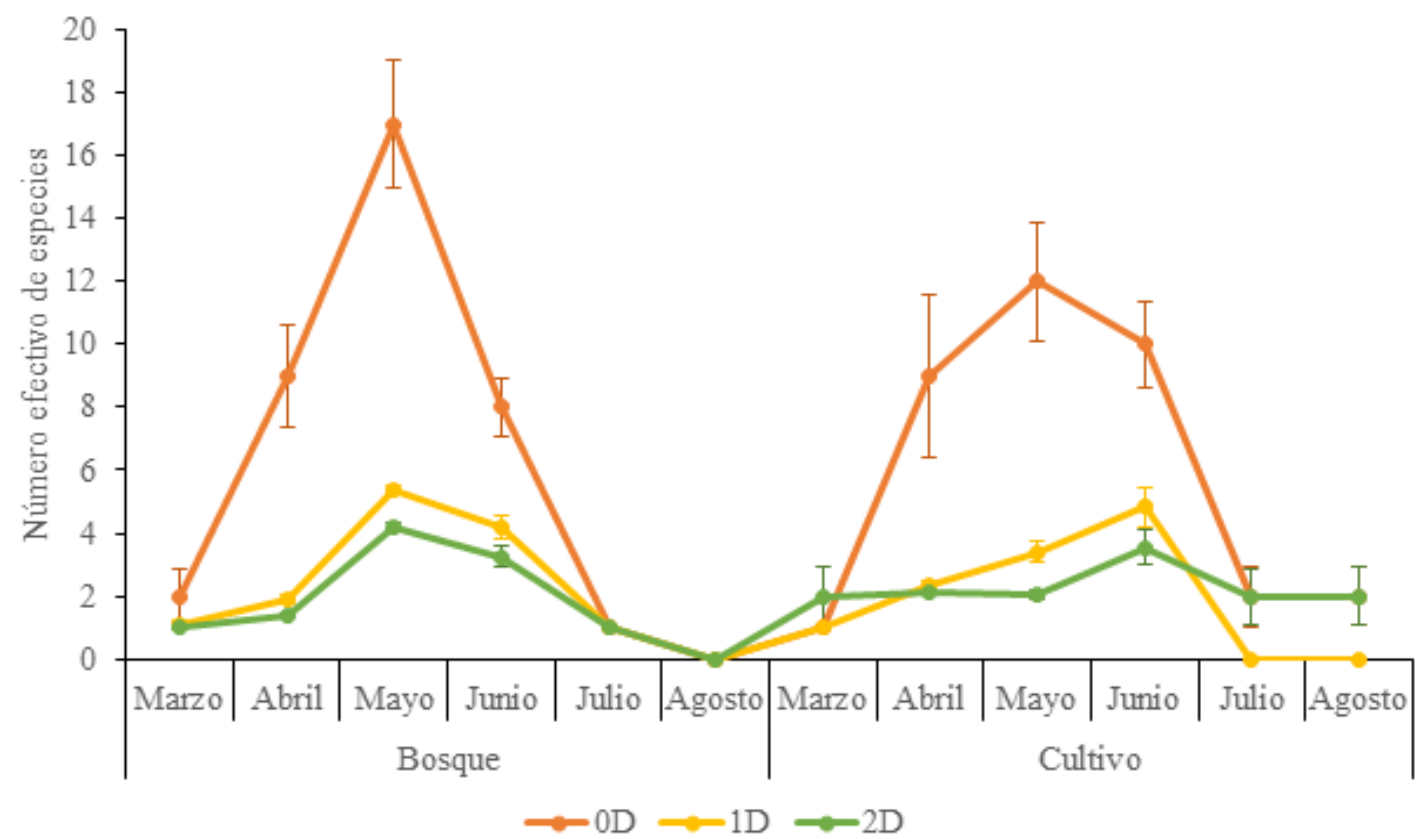

Figura 4. Perfil de diversidad ${ }^{0} D,{ }^{1} D$ y ${ }^{2} D$ de escarabajos fitófagos (Coleoptera: Scarabaeidae) recolectados en bosque y cultivo durante los meses de muestreo en la RCM, departamento del Atlántico, Colombia.

recursos a consumir y nuevos microhábitats que le sirven a los herbívoros como refugio ante posibles amenazas de sus depredadores (Huston 1994, Barraza et al. 2010).

Para el cultivo, la tendencia fue similar a excepción de la disimilitud de mayo con junio que fue del $44 \%$. Si bien, la dominancia es mayor en los meses con bajas precipitaciones, especialmente durante marzo en las dos áreas de estudio, dada la alta abundancia de Astaena sp. en bosque y L. quadridens en cultivo. En general, la tendencia en la variación temporal correspondió a que la diversidad beta estuvo más influenciada por la pérdida de especies que por el reemplazo. Esto, por la sincronización de la aparición de especies con el período de máximas lluvias, lo que permitió un aumento en la riqueza de especies en pocos meses de muestreo.

En el bosque la biomasa de gremios por muestreo presentó diferencias significativas $(\mathrm{H}=9,68 ; p=0,03)$, siendo mayo el que presenta la mayor disimilitud con respecto a marzo ( $p$ $=0,05$ ) (Fig. 5). Para el cultivo, no se presentaron diferencias significativas, pero mayo marcó mayor diferencia con respecto a los dos últimos muestreos $(p=0,02)$. Esta alta diferencia con respecto a los otros muestreos obedece a la presencia de las especies más grandes muestreadas en toda la RCM, y al- 
gunas de ellas exclusivas de ese mes y de bajo porte, como $P$. polita recolectada sólo en el bosque y $X$. anomala con bajas abundancias en cultivo. Estas especies conforman el gremio XF. Aunque los dos sitios muestreados presentan el mismo patrón, en el bosque los cambios de la biomasa durante los meses de muestreo son más abruptos, lo que demuestra una mayor sensibilidad de la comunidad de insectos fitófagos de la familia Scarabaeidae a los fuertes cambios ambientales que se presentaron durante el año de muestreo.

En ambos sitios, los gremios presentaron una correlación positiva con algunas variables ambientales, siendo la precipitación la más importante en términos de influencia en la fe- nología (Tabla 3). Por su papel como degradadores primarios y secundarios, los gremios de Scarabaeidae fitófagos pueden ser bioindicadores de ecosistemas importantes para la conservación (Morón et al. 1997; Deloya et al. 2007), considerando la diversidad de éstos y relacionándolos con la variedad de recursos disponibles.

Teniendo en cuenta que la composición gremial de escarabajos (Scarabaeidae) fitófagos es similar en bosques y cultivos, se infiere una baja oferta de recursos durante el tiempo de muestreo por las modificaciones del paisaje y las características intrínsecas del ecosistema que afectan la sincronía de algunas especies de esta familia con la aparición de recursos

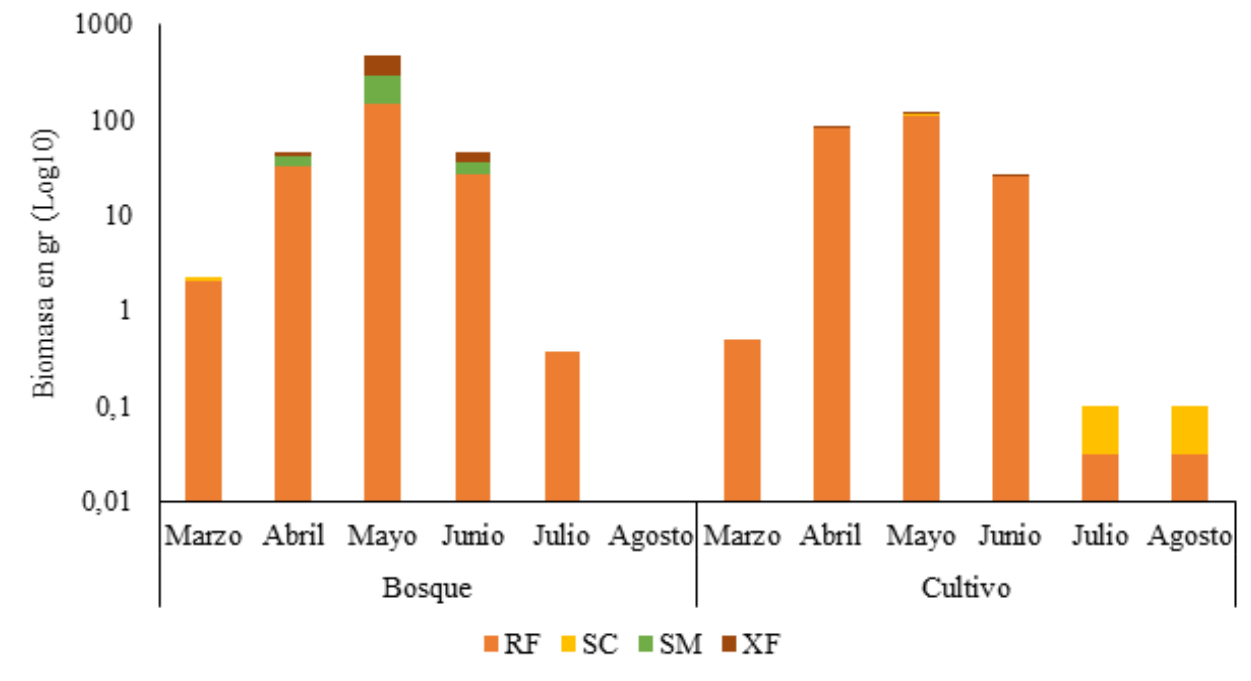

Figura 5. Variación de la biomasa por gremios de escarabajos fitófagos (Coleoptera: Scarabaeidae) en la RCM, departamento del Atlántico. Sm, Sapro-Melífagos; RF, Rizo-Filófagos; SC, Sapro-Caulófagos; XF, Xilo-Filófagos.

temporales en el Bs-T. Lo anterior, genera periodos cada vez más críticos de supervivencia que provocarían la desaparición de especies con nichos restringidos.

Tabla 3. Rangos de correlación de Spearman (valores r) en bosque (b) y cultivo $(C)$. Valores que presentaron significancia $(p>0,05)$ aparecen en negrita.

\begin{tabular}{cccc}
\hline & Temperatura & Humedad & Precipitación \\
\hline Rf-B & $\mathbf{0 , 6 5 7 1 4}$ & $-0,37143$ & $\mathbf{0 , 7 7 1 4 3}$ \\
\hline SM-B & 0,16903 & $\mathbf{- 0 , 8 4 5 1 5}$ & $-0,13522$ \\
\hline SC-B & 0,27045 & 0,30426 & $\mathbf{0 , 7 7 7 5 4}$ \\
\hline XF-B & $\mathbf{0 , 7 7 7 5 4}$ & $\mathbf{- 0 , 5 4 0 9}$ & 0,43948 \\
\hline Rf-C & $\mathbf{0 , 6 6 6 7 4}$ & $\mathbf{- 0 , 5 2 1 7 9}$ & $\mathbf{0 , 6 6 6 7 4}$ \\
\hline SM-C & $\mathbf{0 , 8 2 8 0 8}$ & $\mathbf{- 0 , 6 2 1 0 6}$ & 0,41404 \\
\hline SC-C & 0,18516 & $\mathbf{0 , 5 5 5 4 9}$ & 0,33947 \\
\hline XF-C & 0,45538 & 0,21251 & $\mathbf{0 , 9 4 1 1 2}$ \\
\hline
\end{tabular}

\section{Conclusiones}

La composición del bosque difirió a la del cultivo por tres especies exclusivas del primer sitio, lo que representa un bajo valor de diversidad beta dado por las características similares de los sistemas en cuanto a la disponibilidad de recursos. Las comunidades de escarabajos fitófagos y sus gremios fluctúan en composición, riqueza y abundancia principalmente como respuesta a la estacionalidad climática, más que a la homogeneización de las áreas por el uso agrícola.

En el caso de la biomasa, reflejó que las especies de tallas pequeñas dominaron más en el hábitat del cultivo que en bosque, lo que es una característica propia de sitios intervenidos, y para este caso, por condiciones antropogénicas y naturales. Si se considera que en la costa Caribe colombiana se pueden presentar tres épocas climáticas marcadas se concluye que el 2013 correspondió a un año atípico. Durante el período de muestreo el mayor valor de precipitación correspondió a $99 \mathrm{~mm}$, lo que pudo haber impedido la continuidad en el incremento del número de especies e individuos en la RCM. Adicional a las intervenciones de los ecosistemas por la explotación agrícola y 
ganadera, los tiempos prolongados de sequía pueden repercutir en una posible alta mortalidad de adultos y disminución de la emergencia de pupas.

\section{Literatura citada}

ALCÁZAR-RUIZ, A.; MORÓN-RIOS, A.; MORÓN, M. 2003. Fauna de coleoptera Melolonthidae de Villa las Rosas, Chiapas, México. Acta Zoológica Mexicana (N.S.) 88: 59-86. http://www.scielo.org.mx/scielo.php?script=sci_arttext\&pi$\mathrm{d}=\mathrm{S} 0065-17372003000100003 \& \operatorname{lng}=\mathrm{es} \& \mathrm{tlng}=\mathrm{es}$

ARAGÓN, A.; PÉREZ-TORRES, C. B.; TAPIA, A. M. 2013. Región de Tehuacán. pp. 253-273. En: Morón, M.A.; Aragón, A.; Carrillo Ruíz, H. (Eds.). Fauna de escarabajos del estado de Puebla M.A Morón, Coatepec, Veracruz, México; Coatepec, Veracruz, México.

BARRAZA，J.; MONTES，J.; MARTÍNEZ-HERNÁNDEZ， N.; DELOYA, C. 2010. Ensamblaje de escarabajos coprófagos (Scarabaeidae : Scarabaeinae) del Bosque Tropical Seco, Bahía Concha, Santa Marta (Colombia). Revista Colombiana de Entomología 36 (2): 285-291. http://www.scielo.org.co/pdf/rcen/ v36n2/v36n2a19.pdf

BERNARDI, O.; GARCIA, M.; DA CUNHA, U.; BACK, E.; BERNARDI, D.; RAMIRO, G.; FINKENAUER, E. 2008. Ocorrência de Euetheola humilis (Burmeister) (Coleoptera : Scarabaeidae) em Eucalyptus saligna Smith (Myrtaceae), no Rio Grande do Sul. Neotropical Entomology 37 (1): 100-103. https://dx.doi. org/10.1590/S1519-566X2008000100017

BUSS, E. A. 2006. Flight activity and relative abundance of phytophagous scarabs (Coleoptera: Scarabaeoidea) from two locations in Florida. Florida Entomologist 89 (March): 32-40. https:// doi.org/10.1653/0015-4040(2006)89[32:FAARAO]2.0.CO;2

CARDOSO, P.; RIGAL, F.; CARVALHO, J. C. 2015. BAT - Biodiversity assessment tools, an R package for the measurement and estimation of alpha and beta taxon, phylogenetic and functional diversity. Methods in Ecology and Evolution 6 (2): 232-236. https://doi.org/10.1111/2041-210X.12310

CARVALHO, J. C.; CARDOSO, P.; GOMES, P. 2012. Determining the relative roles of species replacement and species richness differences in generating beta-diversity patterns. Global Ecology and Biogeography 21 (7): 760-771. https://doi.org/10.1111/ j.1466-8238.2011.00694.x

CHAO, A.; JOST, L. 2012. Coverage-based rarefaction and extrapolation: Standardizing samples by completeness rather than size. Ecology 93 (12): 2533-2547. https://doi.org/10.1890/11-1952.1

CLARKE, K. R.; WARWICK, R.; WARWICK, R. M. 2001. An Approach to Statistical Analysis and Interpretation (2nd ed.). PRIMER-E Ltd; UK; 176 p.

COLWELL, R. K.; FUENTES, E. R. 1975. Experimental Studies of the niche. Annual Review of Ecology and Systematics. 6: 33-37.https://www.annualreviews.org/doi/abs/10.1146/annurev. es.06.110175.001433

DELOYA, C.; PARRA-TABLA, V.; DELFÍN-GONZÁLEZ, H. 2007. Fauna de coleópteros Scarabaeidae Laparosticti y Trogidae (Coleoptera: Scarabaeoidea) asociados al Bosque Mesofilo de Montaña, cafetales bajo sombra y comunidades derivadas en el Centro de Veracruz, México. Neotropical Entomology 36 (1): 5-21. https://doi.org/10.1590/S1519-566X2007000100002

ENDRÖDI, S. 1985. Dynastinae of the world. Dr W. Junk Publishers; Budapest; $800 \mathrm{p}$.

ESCALONA, H. E.; JOLY, L. J. 2006. El género Ligyrus en Venezuela. Boletín Sociedad Entomológica Aragonesa (39): 111-137. http://sea-entomologia.org/Publicaciones/PDF/BOLN39/111 137BSEA39Ligyrus.pdf

GARCÍA-AT̄ENCIA, S.; MARTÍNEZ-HERNÁNDEZ, N. 2015. Escarabajos fitófagos (Coleoptera: Scarabaeidae) del departamento del Atlántico, Colombia. Acta Zoológica Mexicana (N.S.) 31 (1): 89-96. https://doi.org/10.21829/azm.2015.311519

GARCÍA-ATENCIA, S.; MARTÍNEZ-HERNÁNDEZ, N.; PAR-
DO-LOCARNO, L. C. 2015. Escarabajos fitófagos (Coleoptera: Scarabaeidae) en un fragmento de bosque seco tropical del departamento del Atlántico, Colombia. Revista Mexicana de Biodiversidad 86 (3): 754-763. https://doi.org/10.1016/j. rmb.2015.07.009

GARCÍA-DE JESÚS, S.; MORENO, C. E.; MORÓN, M. Á.; CASTELLANOS, I.; PAVÓN, N. P. 2016. Integrando la estructura taxonómica en el análisis de la diversidad alfa y beta de los escarabajos Melolonthidae en la Faja Volcánica Transmexicana. Revista Mexicana de Biodiversidad 87 (3): 1033-1044. https:// doi.org/10.1016/j.rmb.2016.06.003

GASCA-ÁLVAREZ, H. J. 2013. New records of Cyclocephala Dejean (Coleoptera: Scarabaeidae: Dynastinae) associated with Caladium bicolor (Aiton) Vent. (Araceae). The Coleopterists Bulletin 67 (4): 416-418. https://doi.org/10.1649/0010065X-67.4.416

GASCA-ÁlVAREZ， H. J.; AMAT-GARCÍA, G. 2010. Synopsis and key to the genera of Dynastinae (Coleoptera, Scarabaeoidea, Scarabaeidae) of Colombia. ZooKeys 34: 153-192. https://doi. org/10.3897/zookeys.34.309

HERNÁNDEZ-CAMACHO, J. 1992. Caracterización geográfica de Colombia. En: Halffter, G. (Ed.), La Diversidad Biológica de Iberoamérica (p. 9) Acta Zoológica Mexicana, Xalapa, Veracruz, México.

HOLDRIDGE, L. R.; GRENKE, W.; HATHEWAY; W.H.; LIANG, T.; TOSI, J.A. 1971. Forest Environments in Tropical Life Zones: A Pilot Study. Pergamon Press, Oxford.

HSIEH TC, MA KH, CHAO A. 2020. iNEXT: Interpolation and Extrapolation for Species Diversity. R package version 2.0.20, http://chao.stat.nthu.edu.tw/wordpress/software_download/.

HUSTON, M. A. 1994. Biological Diversity: The Coexistence of Species on changing landscapes (C. U. Press (Ed.)). Melbourne, Australia; $671 \mathrm{p}$.

IBARRA, M.; DAMBORSKY, M. 2017. Changes in the structure of Melolonthidae (Coleoptera: Scarabaeoidea) assemblages along a temporal gradient in a natural reserve in Chaco, Argentina. Austral Entomology57 (4): 377-386.https://doi.org/10.1111/ aen. 12288

JOLY, L. J.; ESCALONA, H. E. 2010. El género Dyscinetus Harold (Coleoptera: Scarabaeidae: Dynastinae: Cyclocephalini) en Venezuela y la descripción de una nueva especie. Papéis Avulsos de Zoologia. Museu de Zoologia da Universidade de Sao Paulo 50 (14): 203-231. https://doi.org/10.1590/S003110492010001400001

JOST, L. 2006. Entropy and diversity. Oikos 113 (2): 363-375. https://doi.org/10.1111/j.2006.0030-1299.14714.X

LÓPEZ-GARCÍA, M. M.; GARCÍA-ATENCIA, S.; AMAT-GARCÍA, G. 2015a. Escarabajos fitófagos (Coleoptera: Scarabaeidae "pleurosticti") de los Andes Orientales de Colombia (Departamentos de Santander, Boyacá y Cundinamarca). Boletín Científico Museo de Historia Natural de la Universidad de Caldas 19 (2): 322-358. http://www.scielo.org.co/scielo.php?pi$\mathrm{d}=\mathrm{S} 0123-30682015000200020 \&$ script $=$ sci_abstract\&tlng $=\mathrm{es}$

LÓPEZ-GARCÍA, M. M.; GASCA-ALVAREZ, H. J.; AMAT-GARCÍA, G. 2015b. The scarab beetle tribe Pentodontini (Coleoptera: Scarabaeidae: Dynastinae) of Colombia: Taxonomy, natural history, and distribution. Zootaxa 4048 (4): 451-492. https://doi. org/10.11646/zootaxa.4048.4.1

LUGO-GARCÍA, G. A.; ORTEGA-ARENAS, L. D.; GONZÁLEZ-HERNÁNDEZ, H.; ARAGÓN-GARCÍA A.; ROMERO-NÁPOLES J.; RUBIO-CORTÉS R.; MORÓN, M. A. 2011. Melolonthidae nocturnos (Coleoptera) recolectados en la zona agrícola agavera de Jalisco, México. Acta Zoológica Mexicana (n.S.) 27 (2): 341-357. https://doi.org/10.21829/ azm.2011.272757

MARTÍNEZ-HERNÁNDEZ, N.; GARCIA-ATENCIA, S.; GUTIÉRREZ CERPA, M. J.; SANJUÁN-MURILLO, S.; CONTRERAS MEJÍA, C. 2010. Composición y estructura de la fauna de escarabajos (Insecta: Coleoptera) atraídos por trampas de luz en 
La Reserva Ecológica de Luriza, Atlántico, Colombia. Boletín de la Sociedad Entomológica Aragonesa 47: 373-381. http:// sea-entomologia.org/Publicaciones/PDF/BOLN_47/373381BSEA47COMPLETO-45.pdf

MARTÍNEZ, N. J.; FRANZ, N. M.; ACOSTA, J. A. 2009. Structure of the scarab beetle fauna (Coleoptera : Scarabaeoidea ) in forest remnants of western Puerto Rico. Entomotropica 24 (1): 1-9. http://saber.ucv.ve/ojs/index.php/rev_ento/article/view/7483

MARTINO, D. 2007. Deforestación en la Amazonía: principales factores de presión y perspectivas. Revista Del Sur Número 169 3-27. http://www.infobosques.com/descargas/biblioteca/152.pdf

MILET-PINHEIRO, P.; GOMES GONÇALVES, E.; DO AMARAL FERRAZ NAVARRO, D. M.; NUÑEZ-AVELLANEDA, L. A.; MAIA, A. C. D. 2017. Floral scent chemistry and pollination in the Neotropical aroid genus Xanthosoma (Araceae). Flora: Morphology, Distribution, Functional Ecology of Plants 231: 1-10. https://doi.org/10.1016/j.flora.2017.03.006

MORON, M. A. 1985. Los insectos degradadores, un factor poco estudiado en los bosques de México. Folia Entomológica Mexicana 65 (January 1985): 131-137. https://biblat.unam.mx/es/revista/folia-entomologica-mexicana/25

MORÓN, M.A. 1994. Fauna de coleoptera Lamellicornia en las montañas del noreste de Hidalgo, México. Acta Zoológica Mexicana (N.S.) 63: 7-59. https://doi.org/10.21829/azm.1994.63631945

MORÓN, M.; RATCLIFFE, B.; DELOYA, C. 1997. Atlas de los escarabajos de México. Coleoptera Lamellicornia. Vol I Familia Melolonthidae. Subfamilias Rutelinae, Dynastinae, Cetoniinae, Trichiinae, Valginae y Melolonthinae. (1st ed.). Sociedad Mexicana de Entomología, A.C; Xalapa, Veracruz, México; 287 p.

MORÓN, M A. 2004. Escarabajos: 200 millones de años de evolución. Instituto de Ecología A.C; Xalapa, Veracruz, México; 204 p. https://books.google.com.co/books?id=H0UeQwAACAAJ

MORÓN, M.A. 2001. Larvas de escarabajos del suelo en México (Coleoptera: Melolonthidae). Acta Zoológica Mexicana (Nueva Serie) Número especial 1: 111-130. https://azm.ojs.inecol.mx/ index.php/azm/article/view/1848

MORÓN, M.A.; DELOYA, C. 1991. Los coleópteros Lamelicornios de la Reserva de la Biosfera "La Michilia", Durango, México. Folia Entomológica Mexicana 81: 279-283. https://biblat.unam. $\mathrm{mx} / \mathrm{es} /$ revista/folia-entomologica-mexicana/articulo/los-coleopteros-lamelicornios-de-la-reserva-de-la-biosfera-la-michilia-durango-mexico

PARDO-LOCARNO, L.; GONZÁLEZ, J.; RAFAEL PÉREZ, C.; YEPES, F.; FERNÁNDEZ, C. 2012. Escarabajos de importancia agrícola (Coleoptera: Melolonthidae) en la Región Caribe colombiana: Registros y propuestas de manejo. Boletin del Museo Entomológico Francisco Luis Gallego 4 (2): 7-24. https:// ciencias.medellin.unal.edu.co/museos/entomologico/images/ Boletin/2012-12/3.pdf.

PIZANO, C.; GARCÍA, H. 2014. El Bosque Seco Tropical en Colombia. (Vol. 1). Instituto de Investigación de Recursos Biológicos Alexander von Humboldt (IAvH); Bogotá, D.C; 350 p. http:// hdl.handle.net/20.500.11761/9333

R CORE TEAM. 2020. R: A language and environment for statistical computing. R Foundation for Statistical Computing, Vienna, Austria. URL https://www.R-project.org/.

RAMÍREZ-PONCE, A.; MORÓN, M.A. 2009. Relaciones filogenéticas del género Anomala (Coleoptera : Melolonthidae: Rutelinae). Revista Mexicana de Biodiversidad 80: 357-394. https:// doi.org/10.22201/ib.20078706e.2009.002.610

RAMÍREZ-SALINAS, C.; MORÓN, M. A.; CASTRO-RAMÍREZ, A. E. 2004. Descripción de los estados inmaduros de tres especies de Anomala, Ancognatha y Ligyrus (Coleoptera: Melolonthidae: Rutelinae y Dynastinae. Acta Zoológica Mexicana (N.S.) 20 (3): 67-82. http://www.scielo.org.mx/scielo.php?script=sci arttext\&pid=S0065-17372004000300005\&lng=es\&tlng=es

RESTREPO-GIRALDO, H.; MORÓN, M.; VALLEJO, F.; PARDO-LOCARNO, L.; LÓPEZ-ÁVILA, A. 2003. Catálogo de Co- leoptera Melolonthidae (Scarabaeidae pleurosticti) de Colombia. Folia Entomológica de México. 42 (2): 239-263. http://www.folia.socmexent.org/revista/Vol 42/Vol42Num2/239-264.pdf

REYES NOVELO, E.; MORÓN, M.A. 2005. Fauna de Coleoptera Melolonthidae y Passalidae de Tzucacab y Conkal, Yucatán, Mëxico. Acta Zoológica Mexicana (N.S) 21 (2): 15-49. http://www.scielo.org. $\mathrm{mx} /$ scielo.php?script=sci_arttext\&pid=S0065-17372005000200002\&lng=es\&tlng=es

SANABRIA-GARCÍA, R.; GASCA-ÁLVAREZ, H. J.; AMAT-GARCÍA, G. 2012. Sinopsis de la tribu Oryctini (Coleoptera: Scarabaeidae: Dynastinae) de Colombia. Insecta Mundi 0276: 1-64. http://digitalcommons.unl.edu/insectamundi/789

SÁNCHEZ-N, D.; AMAT-GARCÍA, G. 2005. Diversidad de la fauna de artrópodos terrestres en el humedal Jaboque, Bogotá-Colombia. Caldasia 27 (2): 311-329. https://revistas.unal.edu.co/ index.php/cal/article/view/39299

SERRANO, A.; MORÓN, M.A.; BARRO, A.; MOLINA, G. 2017. Comparación entre ensambles de Phyllophaga (Coleoptera : Melolonthidae) del Occidente de Cuba. Revista de Biologia Tropical 65 (1): 351-363. http://dx.doi.org/10.15517/rbt.v65i1.23286

STORK, N. E.; BLACKBURN, T. M. 1993. Abundance, body size and biomass of arthropods in tropical forest. Oikos 67 (3): 483489. https://doi.org/10.2307/3545360

SUÁREZ, G.; AMAT-GARCÍA, G. 2007. Lista de especies de los escarabajos fruteros (Melolonthidae: Cetoniinae) de Colombia. Biota Colombiana 8 (1): 69-76. http://revistas.humboldt.org.co/ index.php/biota/article/view/184/183

ÚTIMA, O.; VALLEJO, L. F. 2010. Escarabajos Melolonthidae (Scarabaeidae-Pleurosticti) de La Montaña Cafetera, departamento de Risaralda, Colombia. Agronómica 16 (2): 31-44.

VALLEJO, L. F.; WOLFF, M. 2013. The genus Phyllophaga Harris (Coleoptera: Scarabaeidae: Melolonthinae) in the Colombian andean mountains. Zootaxa 3722 (2): 101-142. https://doi. org/10.11646/zootaxa.3722.2.1

VILLALOBOS-MORENO, A.; PARDO-LOCARNO, L. C.; CABRERO-SAÑUDO, F. J. 2018. Estacionalidad de escarabajos fitófagos (Coleoptera: Melolonthidae) en un Robledal del Nororiente de los Andes colombianos. Boletin Científico Museo de Historia Natural de la Universidad de Caldas. 22 (1): 163-178. https://revistasojs.ucaldas.edu.co/index.php/boletincientifico/article/view/2749/2544

ZOBEL, M. 1997. The relative role of species pools in determining plant species richness. An alternative explanation of species coexistence? Trends in Ecology \& Evolution 12 (7): 266-269. https://doi.org/10.1016/S0169-5347(97)01096-3

\section{Contribuciones de los autores}

SGA participó en la concepción y diseño del artículo; SGA y GAG definieron el contenido intelectual y la búsqueda de literatura; realizaron los estudios experimentales y la obtención de datos; participaron en el análisis de los datos experimentales; realizaron el análisis estadístico; fueron responsables de preparar el manuscrito y someterlo a publicación.

\section{Conflictos de interés}

Los autores declaramos no tener conflictos de intereses.

\section{Origen y financiación}

La investigación hace parte de la tesis de maestría de la primera autora, la cual recibió financiación otorgada al Grupo Insectos de Colombia mediante la Convocatoria Nacional apoyo para el Fortalecimiento de Grupos de Investigación que soporten programas de posgrado de la Universidad Nacional de Colombia-2012. 